\title{
Ubenimex inhibits cell proliferation, migration and invasion in renal cell carcinoma: The effect is autophagy-associated
}

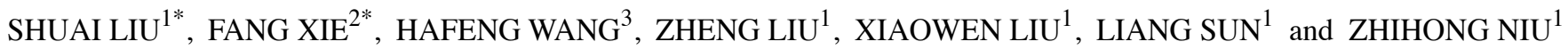 \\ ${ }^{1}$ Department of Urology, Shandong Provincial Hospital Affiliated to Shandong University, Jinan 250021; \\ ${ }^{2}$ Department of Urology, Weihai Municipal Hospital, Weihai, Shandong 264200; \\ ${ }^{3}$ Department of Urology, QiHe People's Hospital, Dezhou, Shandong 251100, P.R. China
}

Received October 22, 2014; Accepted December 12, 2014

DOI: $10.3892 /$ or.2014.3693

\begin{abstract}
Ubenimex is a low-molecular-weight dipeptide with the ability to inhibit aminopeptidase N (APN) activity, enhance the function of immunocompetent cells and confer antitumor effects. We sought to characterize the effects of ubenimex on renal cell carcinoma (RCC). The 786-O and OS-RC-2 human RCC cell lines were positive for APN expression and ubenimex decreased APN activity without affecting the expression. Ubenimex suppressed the proliferation of both cell lines in a concentration-dependent manner, as assessed by curve growth analysis and WST-8 proliferation assay. Wound healing and Matrigel invasion assays demonstrated that the migration and invasion of the RCC cells were also markedly suppressed by ubenimex. Furthermore, ubenimex increased the mortality of both RCC cell lines as determined by the LDH cytotoxicity assay. This affect was accompanied by increased levels of LC3B with no apparent effect on Caspase3; and we observed that autophagy increased significantly after ubenimex treatment in both RCC cell lines by electron microscopy. Moreover, rapamycin enhanced the cytotoxic effect of ubenimex, while 3-methyladenine reversed the effect, indicating that ubenimex cytotoxicity occured through an autophagy-related mechanism. To further assess the potential applicability of ubenimex in the treatment of RCC, we performed immunohistochemistry using tissue microarrays representing $76 \mathrm{RCC}$ patients that underwent radical nephrectomy. The results showed that APN was expressed in most, but not all of the RCC tissues and that the expression was reduced in RCC as compared to
\end{abstract}

Correspondence to: Dr Zhihong Niu, Department of Urology, Shandong Provincial Hospital Affiliated to Shandong University, 324 Jingwu Street, Jinan 250021, P.R. China

E-mail: nzh1789@163.com

*Contributed equally

Key words: renal cell carcinoma, autophagy, ubenimex, proliferation, migration, invasion the normal kidney tissues, suggesting a potential role for APN in RCC development. Collectively, these results indicated that ubenimex inhibits proliferation, migration and invasion of RCC cells. Ubenimex may induce autophagy, which may be associated with its effect on the growth arrest and the cell death of RCC cells.

\section{Introduction}

The treatment of metastatic, locally unresectable renal cell carcinoma (RCC) remains a challenge for urologists; one reason is the poor response rate of the disease to many therapeutic approaches, such as chemotherapy or radiotherapy. Although targeted molecular approaches including vascular endothelial growth factor monoclonal antibodies, tyrosine kinase inhibitors and mammalian target of rapamycin inhibitors that have shown promising results, the overall response rates remain low (1). Thus, drugs targeting new signal transduction pathways are desired.

Ubenimex (Bestatin) is a low-molecular-weight dipeptide molecule that enhances the function of immunocompetent cells and has diverse effects on the production of cytokines. It is also known as an inhibitor of aminopeptidase N (APN), which is identical to the cell surface molecule CD13 (2). APN is involved in various cellular processes, including cell cycle control, cell differentiation and motility, angiogenesis, cellular attachment and invasion/metastasis of various malignancies (3). Previous studies have shown that ubenimex has antitumor activity. It inhibits the invasion of human metastatic tumor cells and induces apoptosis in lung cancer and leukemic cell lines (4-6). In tumor-bearing mice, ubenimex inhibits metastasis or tumor growth and prolongs survival $(7,8)$. In clinical studies, the drug has shown beneficial effects in the treatment of leukemia, non-small cell lung cancer, gastric cancer and cervical cancer (9-12). A potential therapeutic effect of ubenimex in $\mathrm{RCC}$ is suggested by the 5-year remission in a case of residual lymph node metastasis of RCC, following postoperative single administration of ubenimex (13). However, there is little data concerning the mechanism of ubenimex in suppressing tumor cells in RCC. The purpose of the present study was to determine the effects of ubenimex on the proliferation, migration and invasion of RCC cells and the possible mechanism. 


\section{Materials and methods}

Cell culture. The 786-O and OS-RC-2 RCC cell lines were purchased from the cell bank of the Chinese Academy of Sciences. Cells were maintained in RPMI-1640 (HyClone Biotechnology, Carlsbad, CA, USA) supplemented with penicillin, streptomycin and $10 \%$ FBS. The cells were incubated at $37^{\circ} \mathrm{C}$ in a humidified atmosphere with $5 \% \mathrm{CO}_{2}$.

Western blotting. To determine the expression of APN, proteins were extracted from the cells or tissues by suspension in RIPA buffer. Samples were centrifuged at $15,000 \mathrm{rpm}$ at $4^{\circ} \mathrm{C}$ for $10 \mathrm{~min}$ and the supernatants were recovered for analysis. The protein concentrations were determined using the Bradford protein method and the BCA protein assay kit (Sigma, St. Louis, MO, USA). Protein $(40 \mu \mathrm{g})$ was electrophoresed on a pre-cast Bis-Tris polyacrylamide gel $(8 \%)$ and then transferred to a PVDF membrane. The membranes were blotted with rabbit anti-APN (1:1,000; Epitomics Biotechnology, Burlingame, CA, USA), rabbit anti-LC3B (1:1,000; Sigma) rabbit anti-Caspase3 (1:1,000, Abgent; San Diego, CA, USA)or mouse anti-GAPDH (1:3,000 TA08; ZsBio), followed by horseradish peroxidase (HRP)-conjugated secondary antibodies (1:5,000; ZB2306 and ZB2301; both from ZsBio, Beijing, China). Immunoblots were visualized by enhanced chemiluminescence (LAS4000).

Enzyme activity assay. The APN activity in the RCC cells was detected spectrophotometrically using L-leucine-p-nitroanilide (Peptide Institute, Inc., Osaka, Japan) as an APN substrate. Cells $\left(5 \times 10^{4}\right)$ were incubated in a 96 well microtiter plate with $0.1,0.25$ or $0.5 \mathrm{mg} / \mathrm{ml}$ ubenimex at $37^{\circ} \mathrm{C}$ for $24 \mathrm{~h}$. After culture, the medium was aspirated, the cells were washed with PBS and then $200 \mu \mathrm{l}$ of $1 \mathrm{mM}$ alanine-p-nitroanilide was added per well. Each well was then incubated at $37^{\circ} \mathrm{C}$ for $60 \mathrm{~min}$. The APN enzyme activity was estimated by measuring the absorbance at $405 \mathrm{~nm}$ using a microplate reader (Labsystems, Multiskan Bichromatic, Helsinki, Finland).

Growth curve analysis. Cells were trypsinized and $1.0 \times 10^{4}$ cells were plated in individual wells of a 24-well plate containing RPMI-1640 with $10 \%$ FBS. Cells were treated with $0.1,0.25$ or $0.5 \mathrm{mg} / \mathrm{ml}$ ubenimex. Every $24 \mathrm{~h}$, the medium was removed, adherent cells were trypsinized and the total number of adherent cells in each well was quantified using a hematocytometer. The cell counts for 3 wells/time-point were averaged for each group and the data were used to draw growth curves.

WST-8 cell proliferation assay. Cells in an exponential phase of growth were harvested and seeded in 96-well plates at a density of 3,000 cells/well in RPMI-1640 supplemented with different concentrations of ubenimex. After a 24 or $48 \mathrm{~h}$ culture, a $10 \mu \mathrm{l}$ WST- 8 solution (WST- 8 cell proliferation and cytotoxicity assay kit; Dojindo, Kumamoto, Japan) was added into each well. Plates were then incubated for an additional $1 \mathrm{~h}$ at $37^{\circ} \mathrm{C}$ and the absorbance was determined using a microplate reader (EL340 Bio-Tek Instruments, Hopkinton, MA, USA) at $450 \mathrm{~nm}$.

Wound healing migration assays. The RCC cells were plated in 6-well culture plates and grown to $\sim 100 \%$ confluency before scratching with a sterile P200 pipette tip across the monolayer. The cell debris were removed by being washed with PBS and the cells were cultured in RPMI-1640 and 2\% FBS supplemented with different concentrations of ubenimex. The area of the scratch was measured at 0,12 and $18 \mathrm{~h}$ and quantification was performed by measuring the area of cell migration at different time points compared to the scratch area at $0 \mathrm{~h}$. Each experiment was repeated 3 times.

Matrigel invasion assay. Invasion assays were performed using Transwell chambers that were pre-coated with $40 \mu 1$ of $1 \mathrm{mg} / \mathrm{ml}$ Matrigel matrix (BD Bioscience, Bedford, MA, USA). Control untreated cells or cells treated with ubenimex $(0.25 \mathrm{mg} / \mathrm{ml}$ for $24 \mathrm{~h})$ were trypsinized and $1.0 \times 10^{5}$ cells were plated in the upper wells in a serum-free medium, while medium with $10 \%$ FBS was added to the lower well as a stimuli. After $12 \mathrm{~h}$ of incubation, the cells on the Matrigel side of the chambers were removed with a cotton swab. The inserts were fixed in methanol and stained with H\&E staining. The number of the invading cells attached to the other side of the inserts was counted under a light microscope using 8 random fields at x200 magnification. The experiment was performed in triplicate.

LDH cytotoxicity assay. The levels of LDH release were assessed as a method for determining the extent of cell death irrespectively of the type of death. A $200 \mu \mathrm{l}$ volume of cell suspension in complete medium $\left(5 \times 10^{3}\right.$ cells/well) was dispensed in each well of a 96 -well plate. Ubenimex $(0.25$ or $0.5 \mathrm{mg} / \mathrm{ml}), 3-\mathrm{MA}(2 \mathrm{mM})$ and/or rapamycin $(0.1 \mu \mathrm{M})$ was added for $24 \mathrm{~h}$. The 96 -well plates were centrifuged for $5 \mathrm{~min}$ at $400 \mathrm{x} g$ and then $120 \mu \mathrm{l}$ of the supernatant from each well was transferred to a new plate. The plates were incubated at room temperature for $30 \mathrm{~min}$ in the dark and then the absorbance was spectrophotometrically measured at a wavelength of $560 \mathrm{~nm}$.

Electron microscopy. The RCC cells were treated with $0.25 \mathrm{mg} / \mathrm{ml}$ ubenimex for $12 \mathrm{~h}$. They were then fixed with $3 \%$ glutaraldehyde and 2\% paraformaldehyde in a $0.1 \mathrm{M}$ PBS buffer for $30 \mathrm{~min}$, postfixed with $1 \%$ osmium tetroxide for $1.5 \mathrm{~h}$, washed and stained in $3 \%$ aqueous uranyl acetate for $1 \mathrm{~h}$, then dehydrated in an ascending series of ethanol and acetoned and embedded in Araldite. Ultrathin sections were cut on a Reichert ultramicrotome, double stained with $0.3 \%$ lead citrate and examined on a JEOL-1200EX electron microscope (Jeol, Tokyo, Japan).

Human tissues. RCC tissues were obtained from 76 patients diagnosed with RCC (median age, 63.8 years; range, 19-85) who were treated by radical nephrectomy at Shandong Provincial Hospital (Jinan, China) between July 2009 and January 2011. Formalin-fixed and paraffin-embedded specimens were used in our analysis. Ten specimens of non-neoplastic renal tissues were also obtained from these patients. This study was approved by the Ethics Committee of the Shandong Provincial Hospital.

Construction of tissue microarray blocks and immunohistochemistry. Tissue microarrays were constructed using a manual tissue arrayer (Beecher, Silver Spring, MD, USA). Three cylindrical core biopsies $(0.6-\mathrm{mm}$ in diameter) were taken 
A

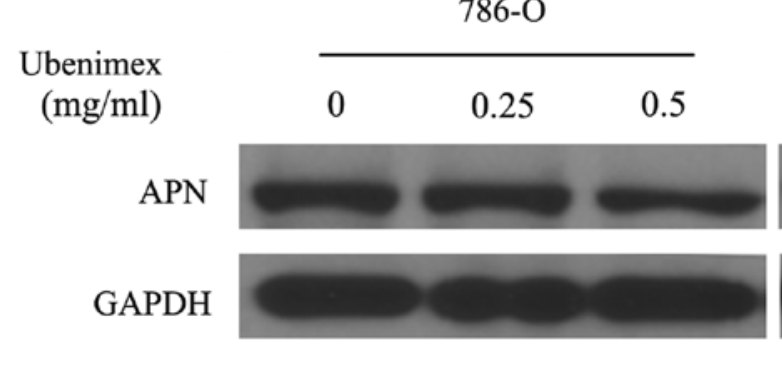

OS-RC-2

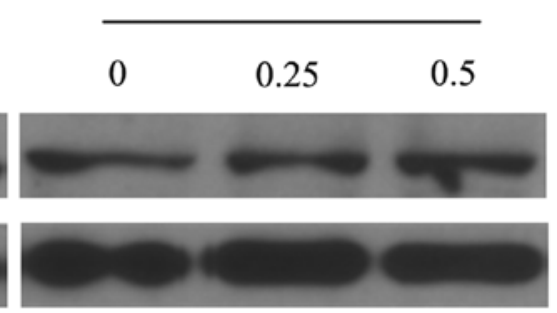

APN enzyme activity assay

B

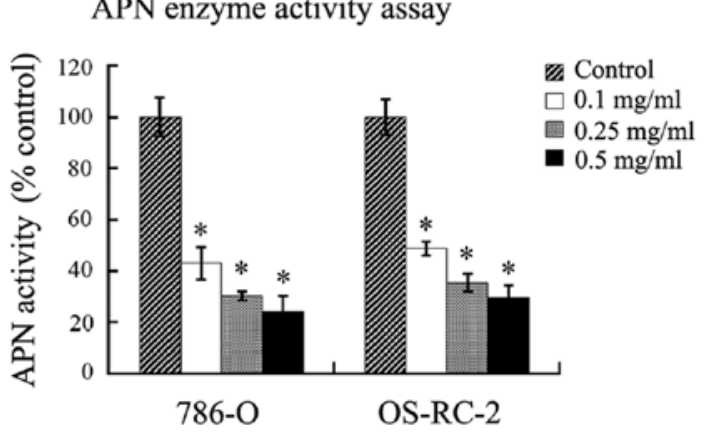

Figure 1. Ubenimex reduces APN activity in the RCC cells. (A) APN expression in the 786-O and OS-RC-2 cells was assessed by western blotting after a 24-h exposure to $0,0.25$ or $0.5 \mathrm{mg} / \mathrm{ml}$ ubenimex. (B) $786-\mathrm{O}$ and OS-RC-2 cells were incubated with ubenimex $(0.1,0.25 \mathrm{or} 0.5 \mathrm{mg} / \mathrm{ml})$ for $24 \mathrm{~h}$. The cells were then washed with PBS twice supplemented with $1 \mathrm{mM}$ alanine-p-nitroanilide for $1 \mathrm{~h}$. Quantification of the APN activity was performed by measuring the absorbance of the supernatant at $405 \mathrm{~nm}$ compared to control untreated cells. Bars indicate the means $\pm \mathrm{SD}$ of 3 independent experiments. ${ }^{*} \mathrm{P}<0.05 \mathrm{vs}$. control untreated cells. APN, aminopeptidase N; RCC, renal cell carcinoma.

from different sites of each tumor and precisely arrayed using a recipient paraffin tissue microarray block. Ten specimens of the non-neoplastic renal tissues were also resected from adjacent regions of the RCCs and analyzed for comparison. To assess APN expression, 4- $\mu \mathrm{m}$ tissue microarray sections were deparaffinized, rehydrated and subsequently incubated with monoclonal rabbit antibodies targeting human APN (1:500; Epitomics Biotechnology). Hematoxylin served as a counterstain. Incubation without the primary antibody was used as a negative control. The expression of APN was evaluated by two independent assessors at x200 magnification and scored as follows: -, negative; + , weak; ++ , moderate and +++ , strong.

Statistical analysis. Data were analyzed statistically by the Student's t-test, $\chi^{2}$ test or Fisher's exact test and analysis was performed using the Statistical Package for Social Science (SPSS for Windows package release 10.0; SPSS Inc., Chicago, IL, USA). $\mathrm{P}<0.05$ was considered statistically significant.

\section{Results}

Ubenimex inhibits APN activity in RCC cells without reducing expression. Ubenimex is known as an inhibitor of APN, which is identical to the cell surface molecule CD13 (2). To confirm the expression of APN in two RCC cell lines, 786-O and OS-RC-2, and to evaluate the effects of ubenimex on APN expression and activity, we performed western blotting and enzymatic activity assays. Both cell lines expressed APN, although expression appeared to be consistently higher in the 786-O cells. Furthermore, the expression remained high after a 24-h treatment with ubenimex (Fig. 1A). Conversely, APN activity was reduced by ubenimex in a dose-dependent manner (Fig. 1B). These results verify that APN is expressed in the RCC cells and suggest that ubenimex targets the activity, but not the expression of APN.

Ubenimex inhibits proliferation, migration and invasion of the RCC cell lines. To examine the effects of ubenimex on the proliferation of RCC cells, the 786-O and OS-RC cells were treated with a range of concentrations of ubenimex and the cell growth was assessed over a 6-day time course. The cell growth was significantly decreased for both the cell lines in a concentration-dependent manner, although the effect was more obvious in the 786-O cells (Fig. 2A). These results were verified by the WST- 8 assay after a $24-$ and 48 -h exposure to ubenimex (Fig. 2B).

To determine whether ubenimex affects the migratory ability of RCC cells, we performed scratch wound-healing migration assays. The migratory abilities of the 786-O and OS-RC-2 cells were significantly suppressed by ubenimex in a concentration-dependent manner after a 12- or 18-h exposure (Fig. 3). We further examined the effect of ubenimex on the invasion activity of RCC cells using Matrigel invasion assays. Pretreatment of ubenimex markedly inhibited the invasive abilities of the 786-O and OS-RC-2 cells, although once again, the effects appeared more dramatic in the 786-O cells (Fig. 4). Collectively, these results suggest that ubenimex inhibits the tumorigenic properties of RCC cells, including proliferation, migration and invasion of RCC cells, although the extent of the effect may vary according to the RCC cell line.

Ubenimex induces autophagic death of RCC cells. To determine whether ubenimex induces the cell death of RCC cells, we performed LDH cytotoxicity assays following ubenimex 
A

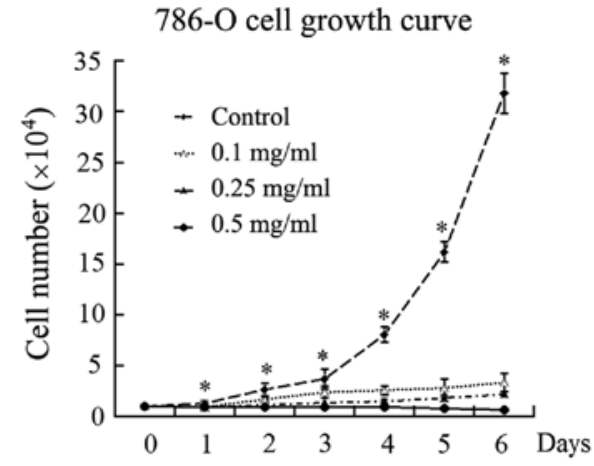

B

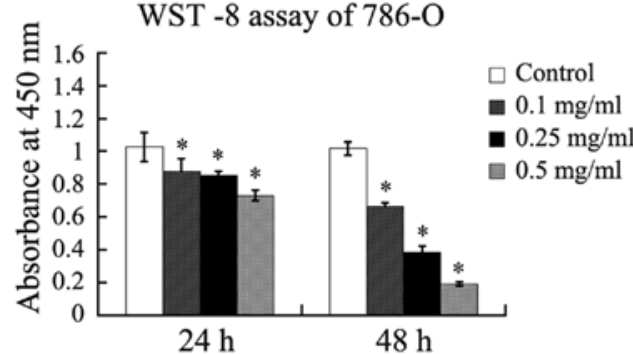

OS-RC-2 cell growth curve
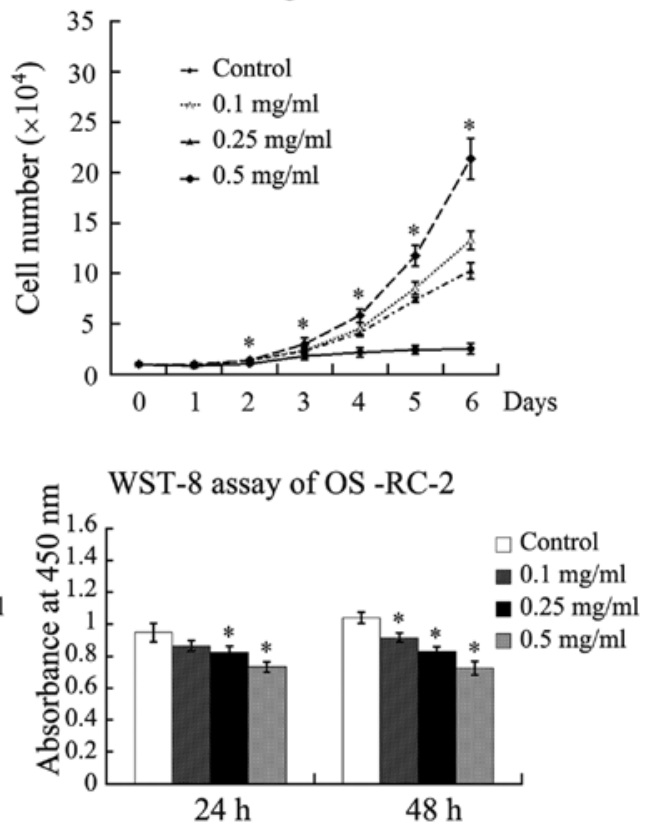

$24 \mathrm{~h}$

$48 \mathrm{~h}$

Figure 2. Ubenimex inhibits the proliferation of the RCC cells. (A) The growth curves of RCC cell lines 786-O and OS-RC-2 were generated after plating $1.0 \times 10^{4}$ cells in triplicate wells. The cell numbers were calculated by the hemocytometry of trypsinized cells over a 6 -day timecourse of culture with the indicated concentrations of ubenimex. Data represents the means $\pm \mathrm{SD}$ of 3 independent experiments. ${ }^{*} \mathrm{P}<0.05$ for control untreated cells compared to all the doses of ubenimex. (B) WST-8 proliferation assay was performed after a 24 ' or 48 -h culture of the 786-O or OS-RC2 cells with the indicated concentrations of ubenimex. Data are shown as the means \pm SD of 3 independent experiments. ${ }^{*}<0.05$ vs. control untreated cells. RCC, renal cell carcinoma.
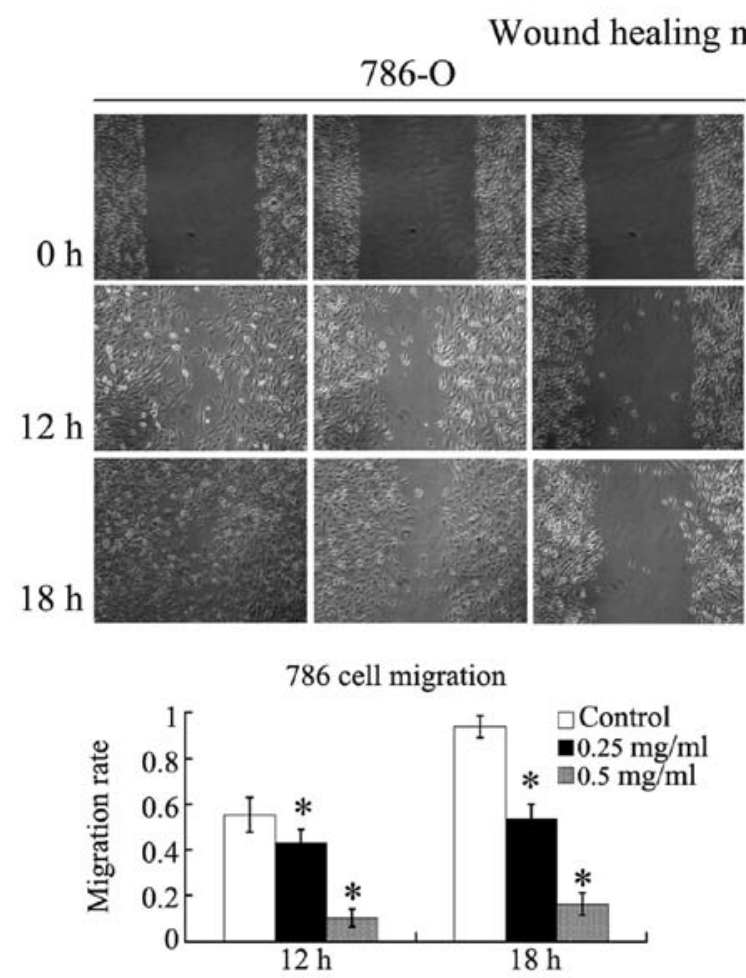
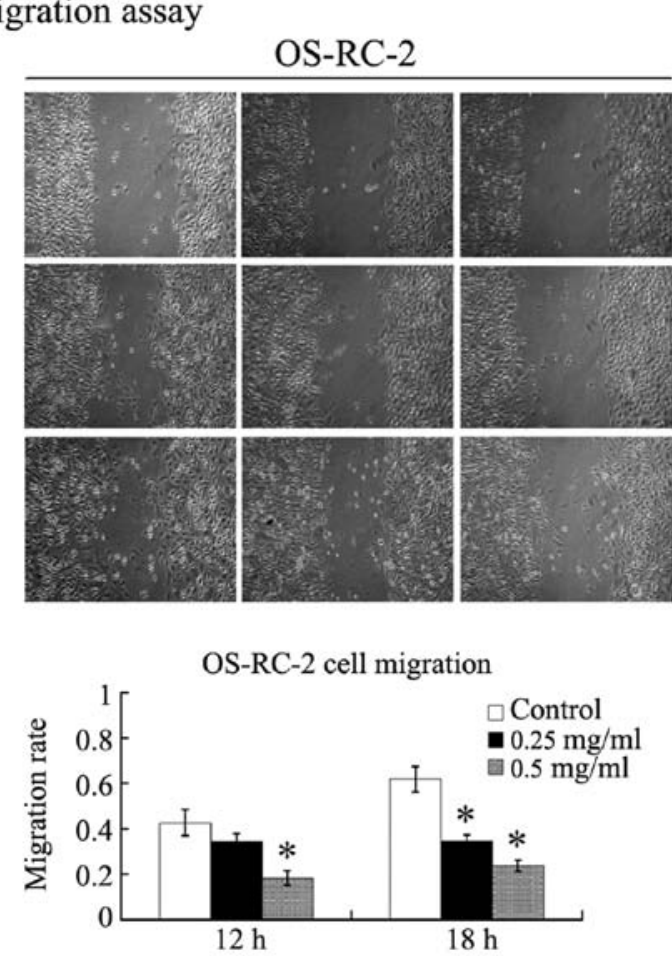

Figure 3. Ubenimex reduces the RCC cell migration ability. Wound healing migration assays were performed to determine the motility of the 786-O and OS-RC-2 cells. Images of representative wells are shown after the indicated times of culture in control medium or medium with 0.25 or 0.5 mg/ml ubenimex (top panel). Quantification was performed by measuring the area migrated at different time points compared to the scratch area at $0 \mathrm{~h}$ (bottom panel). Values represent the means $\pm \mathrm{SD}$ of 3 independent experiments. $\mathrm{P}<0.05$ vs. control untreated cells. RCC, renal cell carcinoma.

treatment. The LDH assay determines the extent of cell death irrespectively of the type of cell death. The mortality of both the RCC cells was significantly increased after treatment with $0.5 \mathrm{mg} / \mathrm{ml}$ ubenimex for 18 or $24 \mathrm{~h}$; the mortality of the 
786-O

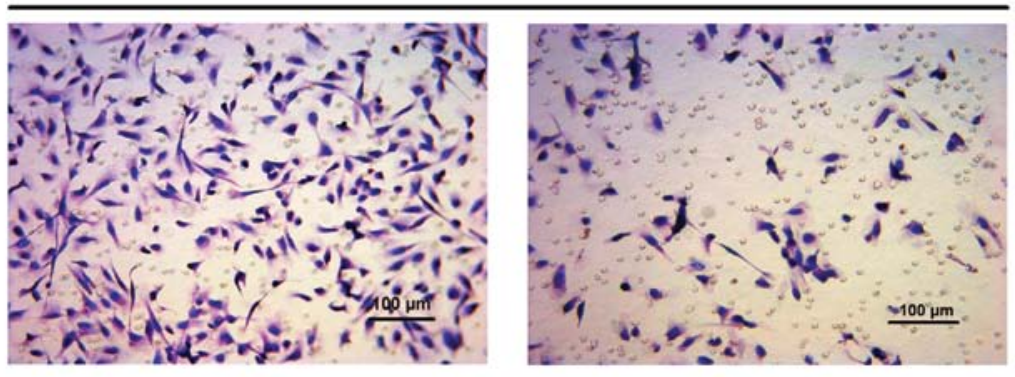

OS-RC-2

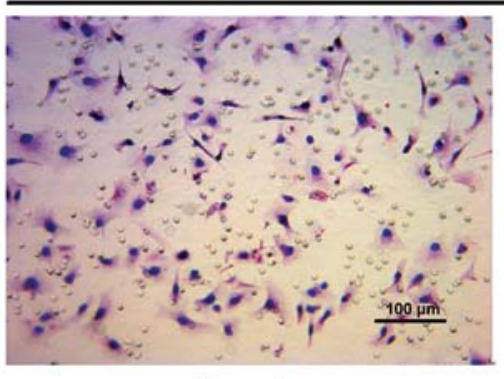

Control

Cell invasion assay of 786-O

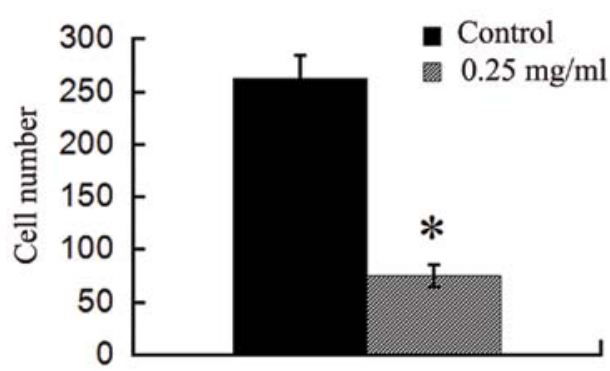

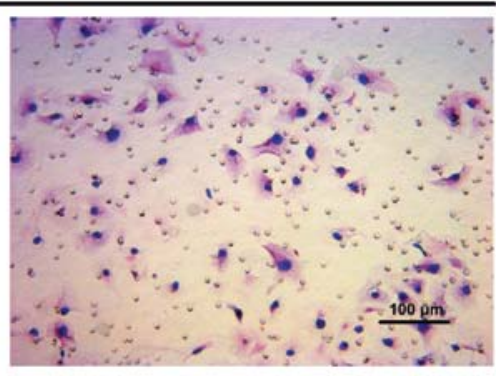

$0.25 \mathrm{mg} / \mathrm{ml}$ Ubenimex

Cell invasion assay of OS-RC-2

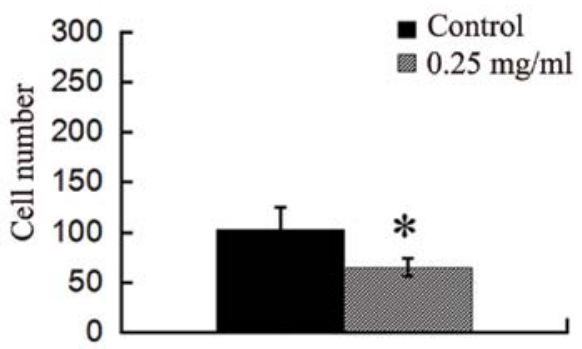

Figure 4. Ubenimex reduces the RCC cell invasive ability. Invasion assays were performed using Matrigel-coated Transwell chambers. The cells were pretreated with ubenimex $(0.25 \mathrm{mg} / \mathrm{ml})$ or left untreated (control; culture medium only) for $24 \mathrm{~h}$ and then trypsinized and plated in the upper wells in a serum-free medium at a concentration of $1 \times 10^{5} /$ well. After $12 \mathrm{~h}$, the numbers of cells invading the Matrigel and migrating to the lower wells were counted following H\&E staining. The left panels show representative images of the lower wells. The right panels show the means \pm SD of the cell numbers in 8 random fields of the 3 independent experiments. ${ }^{*} \mathrm{P}<0.05$ vs. control untreated cells. RCC, renal cell carcinoma.

786-O cells was also significantly increased after treatment with $0.25 \mathrm{mg} / \mathrm{ml}$ ubenimex for 18 or $24 \mathrm{~h}$ (Fig. 5).

To investigate the mechanism of this effect, we assessed the levels of an apoptosis marker (Caspase3) and an autophagy marker (LC-3B). LC-3B expression increased after ubenimex treatment, while the expression of Caspase 3 remained unchanged (Fig. 6A) These results indicated that autophagic cell death, rather than apoptotic cell death may be the predominate mode of ubenimex-induced RCC cell cytotoxicity. To verify this hypothesis, an LDH cytotoxicity assay was performed after pretreating the RCC cells with rapamycin (an inducer of autophagy) or 3-methyladenine (an inhibitor of autophagy). Rapamycin enhanced the levels of ubenimex-induced cell death while 3-methyladenine reversed the effect in both cell lines (Fig. 6B). Western blotting confirmed the relationship between ubenimex and autophagy in 786-O cells (Fig. 6C). These results indicate that ubenimex promotes significant levels of cell death in RCC cells and that the death occurs via an autophagic mechanism.
In order to confirm this theory, electron microscopy was used to visualize the cell morphology after both cell lines were treated with ubenimex compared to the control cells. Ubenimex treatment increased the presence of autophagosomes filled with debris in both the cell lines; only a few vacuoles were observed in the control cells (Fig. 7A). Quantification of the autophagosomes indicated higher autophagy levels after ubenimex treatment (Fig. 7B).

APN is expressed in patient RCC tissues at reduced levels. Immunohistochemical analysis showed strong expression of APN in the epithelial cells of the renal proximal tubules of the normal kidney tissue. Most of the RCC tumor tissues (59/76, 77.6\%) also showed positive staining, although the staining was comparably weak (Fig. 8A and B). Reduced expression of APN in the RCC tissue was verified by western blotting of 4 representative normal adjacent kidney tissues/RCC tissue pairs (Fig. 8C). The intensity of APN was not significantly different between the clear cells and 
Table I. Immunohistochemistry of APN in RCC tissue microarray blocks.

\begin{tabular}{|c|c|c|c|c|c|c|}
\hline & \multirow[b]{2}{*}{ No. of patients } & \multicolumn{4}{|c|}{ Intensity of APN expression } & \multirow[b]{2}{*}{ P-value } \\
\hline & & - & + & ++ & +++ & \\
\hline \multicolumn{7}{|c|}{ Histological type } \\
\hline Clear cell & 67 & 15 & 37 & 15 & - & \\
\hline Papillary & 9 & 2 & 4 & 3 & - & 0.576 \\
\hline \multicolumn{7}{|l|}{ Tumor grade } \\
\hline 1 & 10 & 1 & 8 & 1 & - & \\
\hline 2 & 54 & 14 & 27 & 13 & - & \\
\hline 3,4 & 12 & 2 & 6 & 4 & - & 0.264 \\
\hline \multicolumn{7}{|l|}{ Tumor stage } \\
\hline 1 & 40 & 9 & 19 & 10 & - & \\
\hline 2 & 18 & 5 & 11 & 2 & - & \\
\hline 3,4 & 18 & 3 & 11 & 4 & - & 0.565 \\
\hline \multicolumn{7}{|c|}{ Tumor diameter $(\mathrm{cm})$} \\
\hline$\leq 4$ & 21 & 4 & 11 & 6 & - & \\
\hline $\begin{array}{l}>4 \text { and } \leq 7 \\
>7\end{array}$ & 28 & 9 & 14 & 5 & - & \\
\hline
\end{tabular}

The intensity of APN was not significantly different between clear cell and papillary RCC and was not associated with tumor size, grade or stage. APN, aminopeptidase N; RCC, renal cell carcinoma.

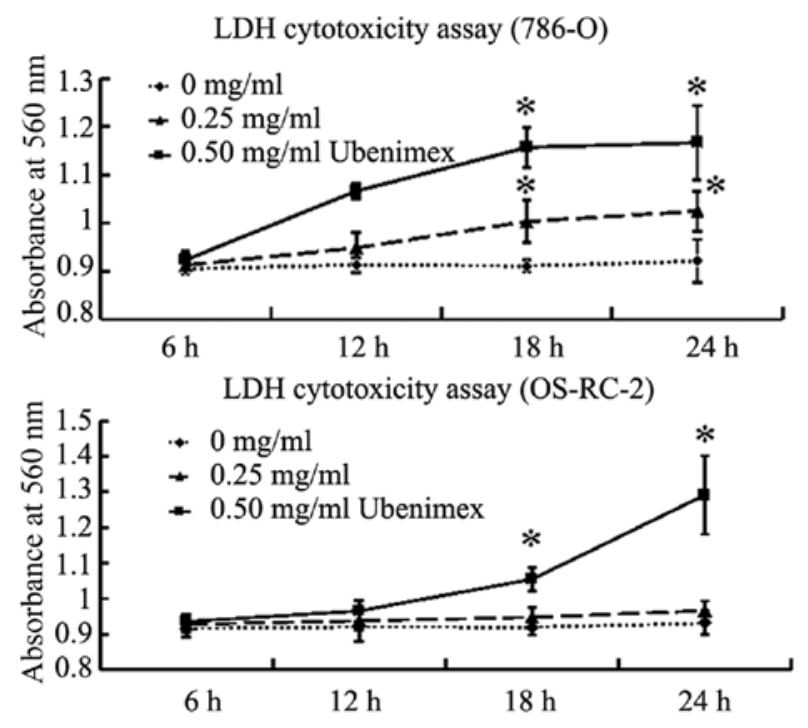

Figure 5. Cytotoxicity of ubenimex. The cytotoxicity of 786-O and OS-RC-2 cells was assessed by an LDH assay after different time course exposure to ubenimex at 0.25 or $0.5 \mathrm{mg} / \mathrm{ml}$. Results represent the means $\pm \mathrm{SD}$ of triplicate wells.

the papillary RCC and was not associated with tumor size grade or stage (Table I). These results suggest that APN was expressed in the RCC, but the levels of expression was reduced as compared to the normal tissues. The differential expression of APN may be suggestive of a function of this protein in RCC. Furthermore, the expression of APN in some RCC tumors provides a rationale for considering ubenimex for the treatment of those tumors that express APN, although the extent of expression may limit the efficacy.

\section{Discussion}

Previous studies indicate that APN plays an important role in the control of the growth and differentiation of cancer cells (3). Inhibition of APN expression or activity reduces the proliferation of various types of cells (2). Here, we gained insight into the effect of ubenimex treatment and the role of APN activity in the growth and development of RCC cells.

Our data demonstrated that treatment of the 786-O and OS-RC-2 cells with ubenimex had no effect on the APN expression level, but decreased the APN enzyme activity. We also demonstrated that ubenimex treatment significantly inhibited the RCC cell growth, proliferation, migration and invasion, which may explain its antitumorigenic properties. The effect of ubenimex was more pronounced in the 786-O cells, which express higher amounts of APN. These results suggest that the inhibitory effects of ubenimex may be associated with its ability to block APN enzyme activity.

The role of APN in the cell motility and metastasis of cancer cells is suggested to involve the degradation of neuropeptides, cytokines and immunomodulatory peptides, as well as angiotensins (6). Inhibition of APN suppresses the progressive potential in many malignant solid tumor cells $(7,14-16)$. The present study demonstrated that ubenimex inhibited RCC cell migration and invasion, which is consistent with the role of APN in these processes. The invasion activity of the RCC cell line SKRC-1, which expresses moderate amounts of the APN, may also be suppressed by ubenimex (17). Thus, these results suggest that the APN may play a role in the metastasis of RCC.

We also showed that ubenimex induced a concentration-dependent cytotoxicity of the RCC cells. Several studies have demonstrated a function of ubenimex in inducing 
A
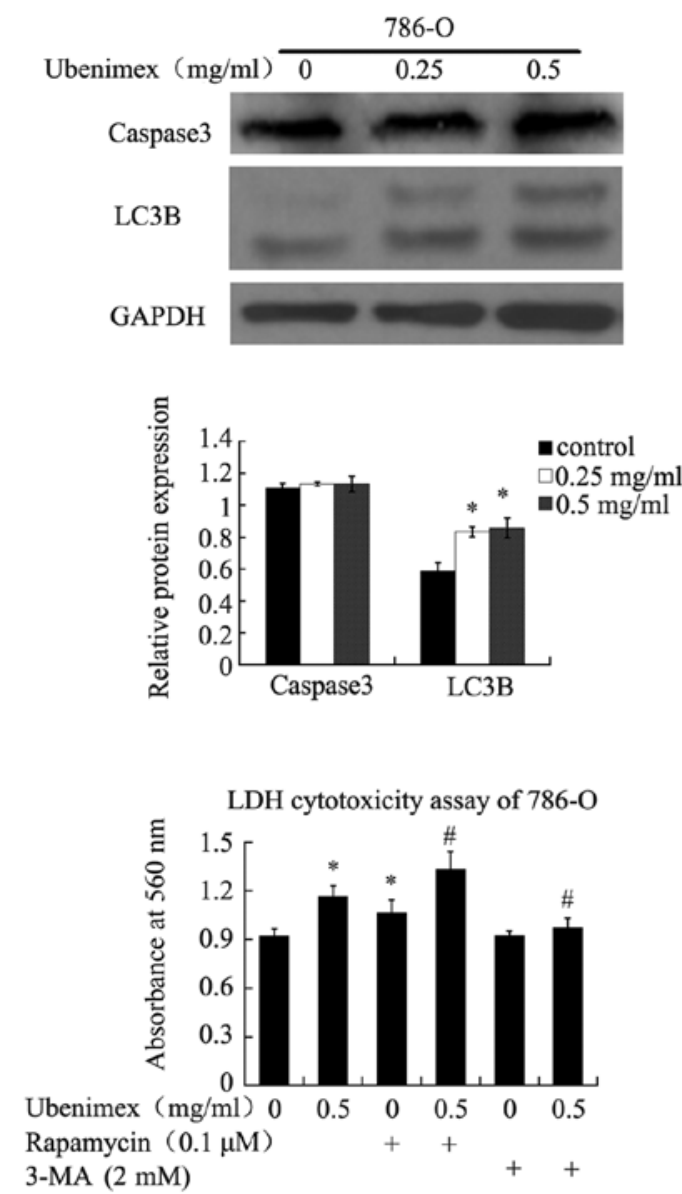
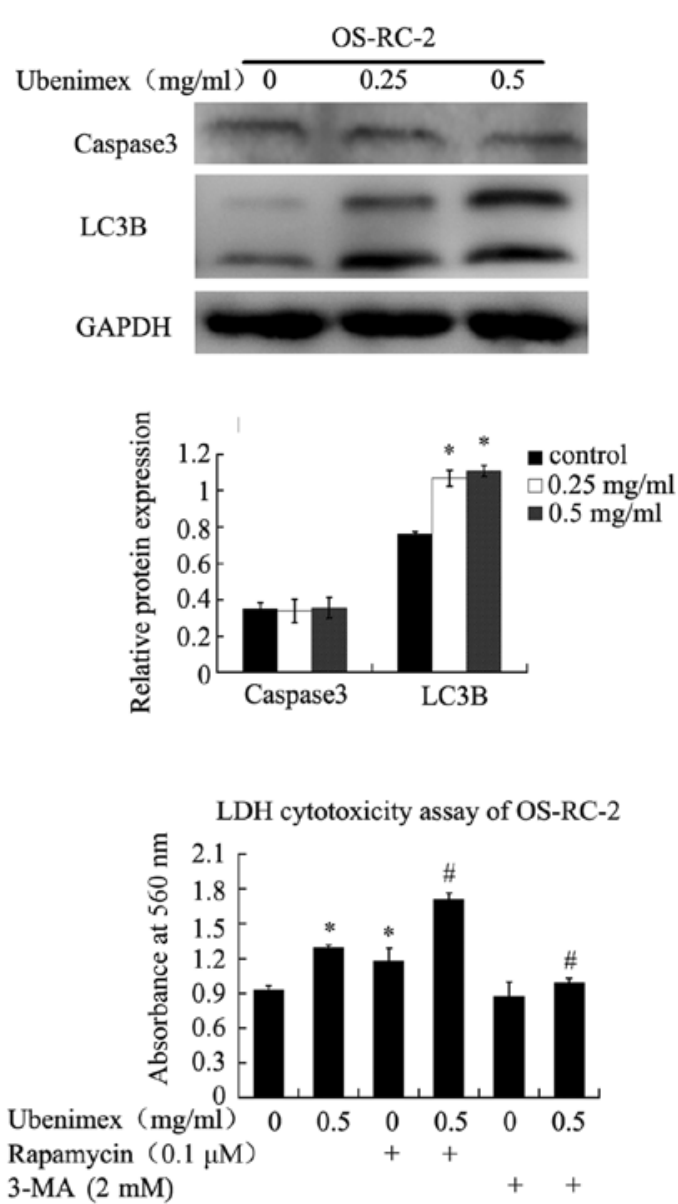

B

Ubenimex $(\mathrm{mg} / \mathrm{ml})$ Rapamycin (0.

C

LC3B

GAPDH

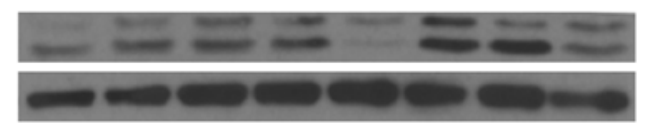

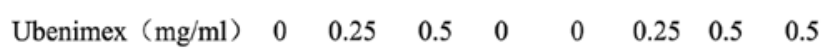

Rapamycin $(0.1 \mu \mathrm{M})$

3-MA (2 mM)

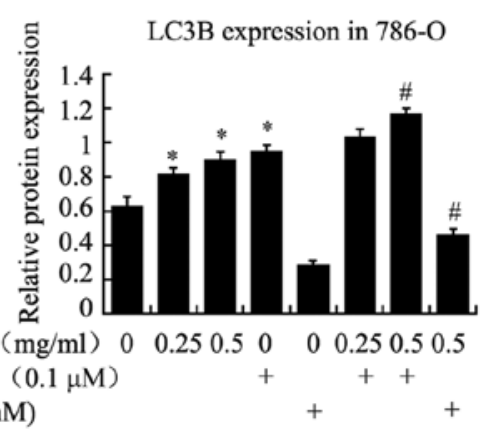

Figure 6. Ubenimex promotes the autophagic death of RCC cells. (A) Western blotting of Caspase 3 and LC-3B expression in 786-O and OS-RC-2 cells after treatment for $24 \mathrm{~h}$ with 0.25 or $0.5 \mathrm{mg} / \mathrm{ml}$ ubenimex. GAPDH is shown as a loading control. Quantification was performed by a densitometry analysis and normalized to GAPDH levels. The bars indicate the means \pm SD from the 3 independent experiments ( $\mathrm{P}<0.05)$. (B). The LDH cytotoxicity assay was performed after a $24 \mathrm{~h}$ treatment of $786-\mathrm{O}$ and $\mathrm{OS}-\mathrm{RC}-2$ cells with ubenimex $(0.5 \mathrm{mg} / \mathrm{ml})$ and/or 3 -methyladenine $(2 \mathrm{mmol} / \mathrm{l})$ or rapamycin $(0.1 \mu \mathrm{mol} / \mathrm{l})$ as indicated. The results represent the means \pm SD from the 3 independent experiments ( ${ }^{*} \mathrm{P}<0.05$ vs. control untreated cells; ${ }^{*} \mathrm{P}<0.05 \mathrm{vs}$. $0.5 \mathrm{mg} / \mathrm{ml}$ ubenimex). (C) $786-\mathrm{O}$ cells were treated as in B. After $12 \mathrm{~h}$, total cell lysates were collected and subjected to western blotting to determine the LC-3B expression (top panel). The intensities of the proteins were quantified by densitometry analysis using Image J software and normalized to GAPDH levels (bottom panel). The bars indicate the means $\pm \mathrm{SD}$ of the 3 independent experiments ( $\mathrm{P}<0.05$ vs. control untreated cells; ${ }^{\mathrm{P}} \mathrm{P}<0.05 \mathrm{vs} .0 .5 \mathrm{mg} / \mathrm{ml}$ ubenimex). RCC, renal cell carcinoma.

cancer cell apoptosis through activating Caspase3 $(11,18,19)$; however, in the present study, ubenimex had no significant effect on the Caspase 3 expression. Instead, LC-3B, which is a key protein marker of autophagy-dependent cell death (20), was upregulated after ubenimex treatment; the cytotoxic effect of ubenimex was attenuated after blocking autophagy with 3-MA, indicating that ubenimex induces the autophagic cell death of RCC cells; furthermore, the autophagy occured after ubenimex treatment in both RCC lines, as evidenced by electron microscopy. To our knowledge, this is the first demonstration that ubenimex induces the autophagy in RCC cells. Autophagy plays a significant role in tumorigenesis and it is the basis of alternative trials evaluating the effectiveness of other drug regimes (20-23). In many cases, the expression of autophagy-related genes in cancer cells can inhibit cell proliferation (24). Reduced levels of autophagy liberate cancer cells from suppression and may further accelerate their proliferation rate $(25,26)$. Therefore, our data are consistent with the possibility that growth inhibition by ubenimex may partly be caused by autophagy. 

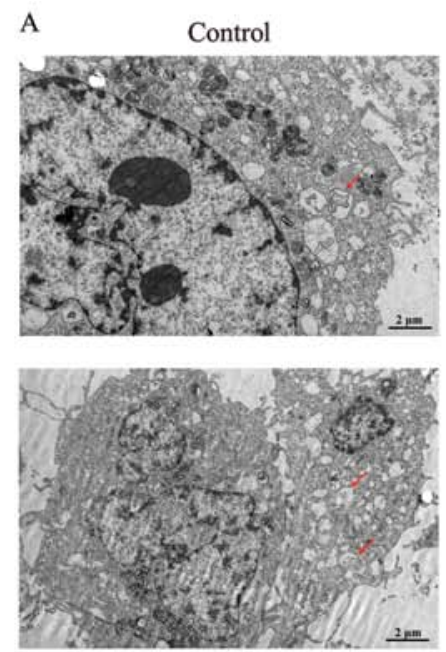

Electron
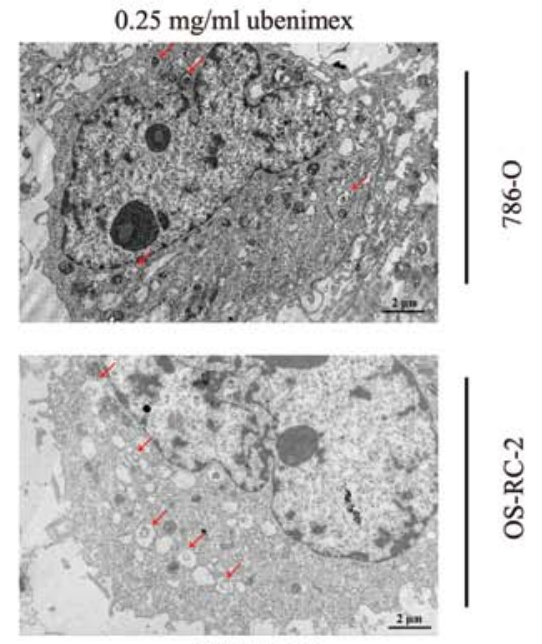

Microscopy

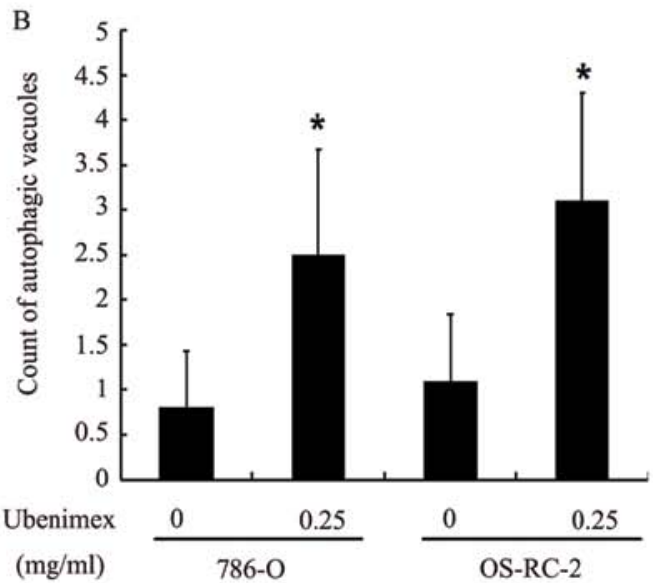

$(\mathrm{mg} / \mathrm{ml}) \quad$ OS-RC-2

Figure 7. Ubenimex treatment increased the presence of autophagosomes filled with debris in both cell lines. (A). RCC 786-O and OS-RC-2 cells were treated with $0.25 \mathrm{mg} / \mathrm{ml}$ ubenimex for $12 \mathrm{~h}$. Then the cells were collected and prepared for electron microscopy analysis. The arrows indicate the appearance of autophagosomes (scale bar, $2 \mu \mathrm{m}$ ). Untreated cells were used as control. (B) Quantification of the autophagosomes in 12 random fields of 3 independent experiments is shown. ${ }^{*} \mathrm{P}<0.05$ vs. control untreated cells. $\mathrm{RCC}$, renal cell carcinoma.

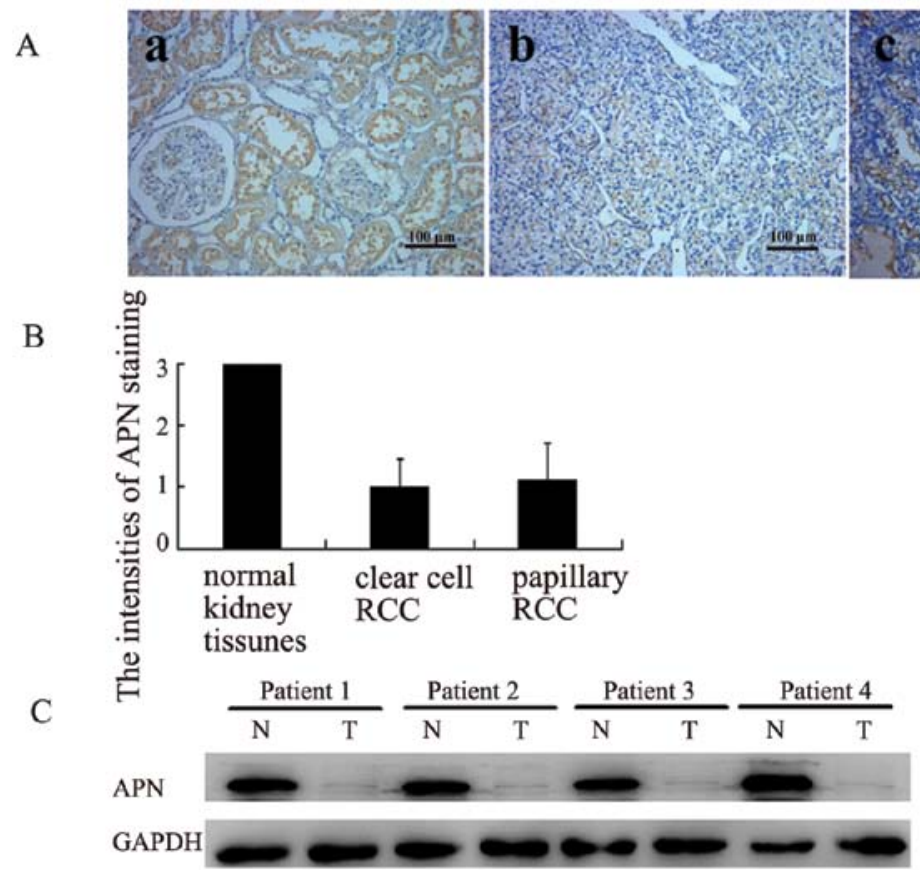

Figure 8. APN is expressed at reduced levels in RCC tissues. (A) Immunohistochemical staining is shown for APN in (a) normal kidney tissue; (b) clear cell RCC; and (c) papillary RCC (x200 magnification). The results are representative of 10 normal kidney tissues, 67 clear cell RCC tissues and 9 papillary RCC tissues. (B) The intensities of APN staining for the samples in (A) were assessed as negative (-, 0 points), weak (+, 1 point), moderate (++, 2 points) or strong $(+++, 3$ points). Values represent the means \pm SD for each sample type. (C) Western blotting of APN expression in RCC tissues (T) and surrounding normal kidney tissues (N). GAPDH was also tested as a loading control. APN, aminopeptidase N; RCC, renal cell carcinoma;

To assess the feasibility of using ubenimex to treat primary human RCC, we also assessed the APN levels in the RCC tissues from patients undergoing nephrectomy. Immunohistochemistry and western blotting showed that APN was expressed in most RCC tumors, but at lower levels than in the surrounding tissues. Although we did not observe any correlations between APN expression and the clinicopathological parameters, downregulation of APN in the tumor tissues compared with normal kidney tissues may suggest a potential role for APN in RCC development. Studies concerning several other cancer types indicate that APN functions in a highly cell type- and context-specific manner: high expression is an adverse prognostic factor for non-small cell lung cancer, pancreatic and colon cancer (27-29), but low APN expression is associated with aggressive disease in meningioma, gastric cancer and prostate cancer $(17,30,31)$. Further studies are 
needed to determine the physiological effects of APN downregulation in RCC and the potential efficacy of the ubenimex in treating RCC.

In conclusion, the present study demonstrated that ubenimex inhibits proliferation, migration and invasion of RCC cells. Ubenimex may induce autophagy, which may partly explain its effect on growth arrest and cell death. Further studies on APN function and the effect of ubenimex treatment in RCC will provide insight into the mode of action of ubenimex in RCC.

\section{Acknowledgements}

This study was supported by the Science and Technology Development Plan Project of Shandong Province, P.R. China.

\section{References}

1. Su D, Stamatakis L, Singer EA and Srinivasan R: Renal cell carcinoma: molecular biology and targeted therapy. Curr Opin Oncol 26: 321-327, 2014.

2. Hitzerd SM, Verbrugge SE, Ossenkoppele G, Jansen G and Peters GJ: Positioning of aminopeptidase inhibitors in next generation cancer therapy. Amino Acids 46: 793-808, 2014.

3. Wickström M, Larsson R, Nygren P and Gullbo J: Aminopeptidase $\mathrm{N}$ (CD13) as a target for cancer chemotherapy. Cancer Sci 102: 501-508, 2011.

4. Ishii K, Usui S, Sugimura Y, Yoshida S, Hioki T, Tatematsu M, Yamamoto $\mathrm{H}$ and Hirano $\mathrm{K}$ : Aminopeptidase $\mathrm{N}$ regulated by zinc in human prostate participates in tumor cell invasion. Int J Cancer 92: 49-54, 2001.

5. Fontijn D, Duyndam MC, van Berkel MP, Yuana Y, Shapiro LH, Pinedo HM, Broxterman HJ and Boven E: CD13/Aminopeptidase N overexpression by basic fibroblast growth factor mediates enhanced invasiveness of 1F6 human melanoma cells. Br J Cancer 94: 1627-1636, 2006.

6. Mina-Osorio P: The moonlighting enzyme CD13: old and new functions to target. Trends Mol Med 14: 361-371, 2008.

7. Terauchi M, Kajiyama H, Shibata K, Ino K, Nawa A, Mizutani S and Kikkawa F: Inhibition of APN/CD13 leads to suppressed progressive potential in ovarian carcinoma cells. BMC Cancer 7: $140,2007$.

8. Martin-Padura I, Marighetti P, Agliano A, Colombo F, Larzabal L, Redrado M, Bleau AM, Prior C, Bertolini F and Calvo A: Residual dormant cancer stem-cell foci are responsible for tumor relapse after antiangiogenic metronomic therapy in hepatocellular carcinoma xenografts. Lab Invest 92: 952-966, 2012.

9. Ichinose Y, Genka K, Koike T, Kato H, Watanabe Y, Mori T, Iioka S, Sakuma A and Ohta M: NK421 Lung Cancer Surgery Group: Randomized double-blind placebo-controlled trial of bestatin in patients with resected stage I squamous-cell lung carcinoma. J Natl Cancer Inst 95: 605-610, 2003.

10. Wakita A, Ohtake S, Takada S, Yagasaki F, Komatsu H, Miyazaki Y, Kubo K, Kimura Y, Takeshita A, Adachi Y, Kiyoi H, Yamaguchi T, Yoshida M, Ohnishi K, Miyawaki S, Naoe T, Ueda R and Ohno R: Randomized comparison of fixed-schedule versus response-oriented individualized induction therapy and use of ubenimex during and after consolidation therapy for elderly patients with acute myeloid leukemia: the JALSG GML200 Study. Int J Hematol 96: 84-93, 2012.

11. Tsukamoto H, Shibata K, Kajiyama H, Terauchi M, Nawa A and Kikkawa F: Aminopeptidase N (APN)/CD13 inhibitor, Ubenimex, enhances radiation sensitivity in human cervical cancer. BMC Cancer 8: 74, 2008.

12. Xu JW, Li CG, Huang XE, Li Y and Huo JG: Ubenimex capsule improves general performance and chemotherapy related toxicity in advanced gastric cancer cases. Asian Pac J Cancer Prev 12: 985-987, 2011.

13. Sugano O, Muto A, Kato M and Ono K: A case of renal cell carcinoma with lymph node metastasis keeping remission for five years by adjuvant immunotherapy with ubenimex. Gan To Kagaku Ryoho 30: 1519-1522, 2003. (In Japanese).
14. Saitoh Y, Koizumi K, Minami T, Sekine K, Sakurai H and Saiki I: A derivative of aminopeptidase inhibitor (BE15) has a dual inhibitory effect of invasion and motility on tumor and endothelial cells. Biol Pharm Bull 29: 709-712, 2006.

15. Mawrin C, Wolke C, Haase D, Krüger S, Firsching R, Keilhoff G, Paulus W, Gutmann DH, Lal A and Lendeckel U: Reduced activity of CD13/aminopeptidase N (APN) in aggressive meningiomas is associated with increased levels of SPARC. Brain Pathol 20: 200-210, 2010.

16. Inagaki Y, Tang W, Zhang L, Du G, Xu W and Kokudo N: Novel aminopeptidase $\mathrm{N}$ (APN/CD13) inhibitor 24F can suppress invasion of hepatocellular carcinoma cells as well as angiogenesis. Biosci Trends 4: 56-60, 2010.

17. Ishii K, Usui S, Sugimura Y, Yamamoto H, Yoshikawa K and Hirano K: Inhibition of aminopeptidase N (AP-N) and urokinase-type plasminogen activator (uPA) by zinc suppresses the invasion activity in human urological cancer cells. Biol Pharm Bull 24: 226-230, 2001.

18. Sekine K, Fujii H and Abe F: Induction of apoptosis by bestatin (ubenimex) in human leukemic cell lines. Leukemia 13: 729-734, 1999.

19. Sekine K, Fujii H, Abe F and Nishikawa K: Augmentation of death ligand-induced apoptosis by aminopeptidase inhibitors in human solid tumor cell lines. Int J Cancer 94: 485-491, 2001.

20. Eum KH and Lee M: Crosstalk between autophagy and apoptosis in the regulation of paclitaxel-induced cell death in v-Ha-ras-transformed fibroblasts. Mol Cell Biochem 348: 61-68, 2011.

21. Natsumeda M, Aoki H, Miyahara H, Yajima N, Uzuka T, Toyoshima Y, Kakita A, Takahashi $\mathrm{H}$ and Fujii Y: Induction of autophagy in temozolomide treated malignant gliomas. Neuropathology 31: 486-493, 2011.

22. Evangelisti C, Ricci F, Tazzari P, Tabellini G, Battistelli M, Falcieri E, Chiarini F, Bortul R, Melchionda F, Pagliaro P, Pession A, McCubrey JA and Martelli AM: Targeted inhibition of mTORC1 and mTORC 2 by active-site mTOR inhibitors has cytotoxic effects in T-cell acute lymphoblastic leukemia. Leukemia 25: 781-791, 2011.

23. Crowley LC, Elzinga BM, O'Sullivan GC and McKenna SL: Autophagy induction by Bcr-Abl-expressing cells facilitates their recovery from a targeted or nontargeted treatment. Am J Hematol 86: 38-47, 2011.

24. Liang XH, Jackson S, Seaman M, Brown K, Kempkes B, Hibshoosh $\mathrm{H}$ and Levine B: Induction of autophagy and inhibition of tumorigenesis by beclin 1. Nature 402: 672-676, 1999.

25. Mathew R, Kongara S, Beaudoin B, Karp CM, Bray K, Degenhardt K, Chen G, Jin S and White E: Autophagy suppresses tumor progression by limiting chromosomal instability. Genes Dev 21: 1367-1381, 2007.

26. Karantza-Wadsworth V,Patel S, Kravchuk O, Chen G, Mathew R, Jin $\mathrm{S}$ and White E: Autophagy mitigates metabolic stress and genome damage in mammary tumorigenesis. Genes Dev 21: 1621-1635, 2007.

27. Hashida H, Takabayashi A, Kanai M, Adachi M, Kondo K, Kohno N, Yamaoka Y and Miyake M: Aminopeptidase N is involved in cell motility and angiogenesis: its clinical significance in human colon cancer. Gastroenterology 122: 376-386, 2002.

28. Ikeda N, Nakajima Y, Tokuhara T, Hattori N, Sho M, Kanehiro H and Miyake M: Clinical significance of aminopeptidase N/CD13 expression in human pancreatic carcinoma. Clin Cancer Res 9: 1503-1508, 2003.

29. Tokuhara T, Hattori N, Ishida H, Hirai T, Higashiyama M, Kodama K and Miyake M: Clinical significance of aminopeptidase $\mathrm{N}$ in non-small cell lung cancer. Clin Cancer Res 12: 3971-3978, 2006

30. Kawamura J, Shimada Y, Kitaichi H, Komoto I, Hashimoto Y, Kaganoi J, Miyake M, Yamasaki S, Kondo K and Imamura M: Clinicopathological significance of aminopeptidase N/CD13 expression in human gastric carcinoma. Hepatogastroenterology 54 : 36-40, 2007.

31. Sørensen KD, Abildgaard MO, Haldrup C, Ulhøi BP, Kristensen H, Strand S, Parker C, Høyer S, Borre M and Ørntoft TF: Prognostic significance of aberrantly silenced ANPEP expression in prostate cancer. Br J Cancer 108: 420-428, 2013. 